\title{
The Value of Serial Serum Lactate Measurements in Predicting the Extent of Ischemic Bowel and Outcome of Patients Suffering Acute Mesenteric Ischemia
}

\author{
Peter Studer • Adrian Vaucher • Daniel Candinas • \\ Beat Schnüriger
}

Received: 14 October 2014 / Accepted: 14 January 2015 / Published online: 30 January 2015

(C) 2015 The Society for Surgery of the Alimentary Tract

\begin{abstract}
Background Acute mesenteric ischemia (AMI) is an emergency with a mortality rate up to $50 \%$. Detecting AMI continues to be a major challenge. This study assed the correlation of repeated preoperative serum lactate with bowel necrosis and to identify risk factors for a lethal outcome in patients with AMI.

Methods A retrospective study of 91 patients with clinically and pathologically confirmed AMI from January 2006 to December 2012 was performed.

Results In-hospital mortality rate was $42.9 \%$. Two hundred nine preoperative lactate measurements were analyzed $(2.3 \pm 1.1$ values per patient). Less than or equal to six hours prior to surgery, the mean serum lactate level was significantly higher (4.97 $\pm 4.21 \mathrm{vs.}$ $3.24 \pm 3.05 \mathrm{mmol} / \mathrm{L}, p=0.006)$ and the mean $\mathrm{pH}$ significantly lower $(7.28 \pm 0.12 \mathrm{vs} .7 .37 \pm 0.08, p=0.001)$ compared to $>6 \mathrm{~h}$ before surgery. Thirty-four patients had at least two lactate measurements within $24 \mathrm{~h}$ prior to surgery. In this subgroup, 17 patients (50 \%) exhibited an increase, 17 patients $(50 \%$ ) a decrease in lactate levels. Forward logistic regression analysis showed that length of necrotic bowel and the highest lactate value $24 \mathrm{~h}$ prior to surgery were independent risk factors for mortality $\left(r^{2}=0.329\right)$.

Conclusion The value of serial lactate and $\mathrm{pH}$ measurements to predict the length of necrotic bowel is very limited. Length of necrotic bowel and lactate values are independent risk factors for mortality.
\end{abstract}

Keywords Acutemesenteric ischemia - Serumlactate $\cdot$ Bowel necrosis $\cdot$ Mortality $\cdot$ Risk factors

\section{Introduction}

Acute mesenteric ischemia (AMI) is a life-threatening condition, requiring immediate intervention to prevent severe abdominal complications and death. The reported mortality remains high, exceeding $50 \%{ }^{1}$ AMI accounts for approximately $0.1 \%$ of all hospital admissions, with an increasing incidence in elderly patients. ${ }^{2}$ Increasing the delay in the

Oral presentation at the 15 th European Congress of Trauma and Emergency Surgery, May 24-27, 2014, Frankfurt, Germany

P. Studer $\cdot$ A. Vaucher $\cdot$ D. Candinas $\cdot$ B. Schnüriger $(\bowtie)$

Department of Visceral Surgery and Medicine, Bern University

Hospital, Bern, Switzerland

e-mail: beat.schnuriger@gmail.com diagnostic process leads to a parallel increase in the mortality rate. ${ }^{3}$ Complete disruption of the intestinal blood supply leads to irreversible mucosal ischemic damage within $6 \mathrm{~h}$.

Transmural bowel infarction presents with severe abdominal pain (94\% of patients) and is often accompanied by nausea, vomiting, bloody diarrhea, or grossly melanotic stool. ${ }^{5} \mathrm{~A}$ pain-free interval of 4-6 h after the onset of the symptoms may be encountered and is partly responsible for delays in the patient's presentation and for diagnostic delays. ${ }^{3}$ Detecting AMI remains a challenge and requires a high degree of suspicion, as the findings are nonspecific and there are no specific laboratory parameters.

Today, multidetector computed tomography (CT) scan is the standard diagnostic tool to detect AMI. The sensitivity $(93.3 \%)$ and specificity $(95.9 \%)$ of the CT for the diagnosis of AMI has matched the sensitivity of angiography $(88 \%)$. $^{6.7}$ Besides the patient's history of symptoms, clinical findings, and radiology, patients with AMI usually present with laboratory signs of marked inflammation, such as leukocytosis. In 
addition, metabolic acidosis may be present. All laboratory parameters tested so far have a poor sensitivity and specificity; however, no serial measurements have been analyzed so far. ${ }^{8}$

The aim of this study was to evaluate preoperative serial serum lactate and $\mathrm{pH}$ in patients suffering AMI. In addition, pre, intra, and postoperative patient characteristics were analyzed in order to identify risk factors for a lethal outcome. To the best of our knowledge, the assessment of serial serum lactate measurements and risk factors for lethal outcome in patients with AMI has never been done before.

\section{Materials and Methods}

A retrospective study was performed of patients with AMI confirmed by laparotomy and histology at Bern University Hospital, Switzerland, between March 2006 and December 2012. Patient characteristics were collected using a computerized spreadsheet (Microsoft Access 2003, Microsoft Corporation, Redmond, WA). The collected demographic and preoperative variables were as follows: age, gender, main diagnosis, body mass index (BMI), prior surgical procedures, smoking history, alcohol abuse, drug history (anticoagulants, platelet aggregation inhibitors, statins, diuretics, antihypertensive drugs), blood gas analysis (lactate, $\mathrm{pH}$ ), serum markers (white cell count, $\mathrm{C}$-reactive protein (CRP), history of arteriosclerosis, chronic cardiovascular disease, chronic pulmonary disease, pre-existing hepatological disease, diabetes mellitus, bowel sounds present at admission, clinical signs of peritonitis, onset of abdominal symptoms, preoperative severe sepsis [systemic inflammatory response syndrome (SIRS) + source of infection + organ dysfunction/hypotension or hypoperfusion (lactic acidosis, systolic blood pressure $<90 \mathrm{mmHg}$ or systolic blood pressure drop $\geq 40 \mathrm{mmHg}$ )], and American Society of Anesthesiologists (ASA) score. Intraoperative variables collected included the following: type of surgical procedure performed, length of bowel necrosis, location of necrosis, amount of blood loss, and operation time. Postoperative parameters collected included the following: in-hospital survival, histology, surgical site and systemic complications, intensive care unit length of stay (ICU-LOS), and hospital length of stay (HLOS).

Continuous and categorical variables are reported as mean \pm standard deviation (SD), median \pm range, and percentages. The highest lactate and $\mathrm{pH}$ levels measured within the last $24 \mathrm{~h}$ prior to surgery were correlated to the length of necrotic bowel by linear regression analysis. Mean lactate and $\mathrm{pH}$, stratified according to the time before surgery, were compared using analysis of variance (ANOVA). In order to identify risk factors for fatal outcome in patients with AMI, all parameters were compared between the survivor and nonsurvivor groups. Proportions and continuous variables were compared using the Fisher exact and the Mann-Whitney $U$ tests, respectively. Potential risk factors were identified using $p<0.2$. Moreover, those variables of special interest were forced into the equation. Forward logistic regression analysis was used to identify independent risk factors for mortality.

All statistical analyses were performed using the Statistical Package for Social Sciences (SPSS Windows ${ }^{\circ}$ ), version 17.0 (SPSS Inc., Chicago, IL). $p<0.05$ was considered statistically significant.

\section{Results}

Patient Characteristics

Between March 2006 and December 2012, a total of 91 patients with AMI were identified and included in this study. Demographic characteristics are listed in Table 1. The average age was $66.7 \pm .15 .7$ years old and $58.0 \%(n=49)$ of patients were female. On average, $30.8 \%(n=28)$ of patients were on regular therapy with acetylsalicylic acid, $14.3 \%(n=13)$ were prescribed phenprocoumon, $9.9 \%(n=9)$ clopidogrel, and $54.9 \%(n=50)$ at least one antihypertensive drug. A total of $46.2 \%(n=42)$ of patients were smokers and $18.7 \%(n=17)$ had a history of alcohol abuse. Comorbidities were frequently detected, with $68.1 \%(n=62)$ of patients with known cardiovascular disease and $39.6 \%(n=36)$ with manifest arteriosclerosis. Furthermore, $42.9 \%(n=39)$ had a pre-existing pulmonary disease and $19.8 \%(n=18)$ suffered from diabetes mellitus.

Overall, $24.2 \%(n=20)$ of patients presented with severe sepsis on admission to our service. On clinical examination, $40.7 \%(n=37)$ of patients presented with signs of peritonitis; however, no statistically significant differences between the survivor and the non-survivor groups were found with respect to these two parameters. Of the study population, $82.6 \%(n=$ 78) underwent an i.v. contrast-enhanced computed tomography (CT) scan before laparotomy, which revealed signs of AMI in $25.3 \%(n=23)$ of patients.

\section{Serial Serum Lactate and $\mathrm{pH}$ Values}

A total of 209 preoperative lactate measurements were available for analysis $(2.3 \pm 1.1$ preoperative lactate values per patient). The highest serum lactate value measured within $24 \mathrm{~h}$ before surgery in individual patients showed a moderate correlation, but no statistical significance with the length of bowel necrosis $\left(r^{2}=0.257, p=0.058\right)$.

A significant increase in the mean serum lactate levels ( $4.9 \pm 4.2$ vs. $3.2 \pm 3.1 \mathrm{mmol} / \mathrm{L}, p=0.006)$ and a decrease in the mean serum $\mathrm{pH}(7.2 \pm 0.1$ vs. $7.4 \pm 0.1, p=0.001)$ was detected in the period 0 to $6 \mathrm{~h}$ before surgery, compared to values $>6 \mathrm{~h}$ prior to surgery (Fig. 1). This finding was accentuated for patients with $>50 \mathrm{~cm}$ of necrotic bowel (Table 2). Furthermore, within $24 \mathrm{~h}$ before surgery, the non-survivors 
Table 1 Demographics and patients characteristics
$B M I$ body mass index, $A S A$ American Society of Anesthesiologists, $C T$ computed tomography

\begin{tabular}{lllll}
\hline & All $(n=91)$ & Survivor $(n=52)$ & Non-survivor $(n=39)$ & $p$ \\
\hline Age (years) & $66.9 \pm 15.7$ & $65.3 \pm 16.4$ & $68.0 \pm 15.1$ & 0.209 \\
Gender (male) & $42(46.2 \%)$ & $21(53.8 \%)$ & $21(40.4 \%)$ & 0.212 \\
BMI & $76(26.2 \pm 5.4)$ & $46(26.0 \pm 5.2)$ & $30(26.5 \pm 5.9)$ & 0.815 \\
Smoking & $42(46.2 \%)$ & $27(51.9 \%)$ & $15(38.5 \%)$ & 0.288 \\
Alcohol & $17(18.7 \%)$ & $11(21.2 \%)$ & $6(15.4 \%)$ & 0.591 \\
Aspirin & $28(30.8 \%)$ & $16(30.8 \%)$ & $12(30.8 \%)$ & 1.000 \\
Phenprocoumon & $13(14.3 \%)$ & $9(17.3 \%)$ & $4(10.3 \%)$ & 0.383 \\
Clopidogrel & $9(9.9 \%)$ & $6(11.5 \%)$ & $3(7.7 \%)$ & 0.727 \\
Statin & $24(26.4 \%)$ & $13(25.0 \%)$ & $11(28.2 \%)$ & 0.812 \\
Antihypertensives & $50(54.9 \%)$ & $30(57.7 \%)$ & $20(51.3 \%)$ & 0.671 \\
Arteriosclerosis & $36(39.6 \%)$ & $23(44.2 \%)$ & $13(33.3 \%)$ & 0.387 \\
Cardiovascular disease & $62(68.1 \%)$ & $36(69.1 \%)$ & $26(66.7 \%)$ & 0.823 \\
Pulmonary disease & $39(42.9 \%)$ & $19(36.5 \%)$ & $20(51.3 \%)$ & 0.201 \\
Nephrological diseases & $43(47.3 \%)$ & $22(42.3 \%)$ & $21(53.8 \%)$ & 0.297 \\
Diabetes mellitus & $18(19.8 \%)$ & $7(13.5 \%)$ & $11(28.2 \%)$ & 0.111 \\
Hepatological diseases & $11(12.1 \%)$ & $5(9.6 \%)$ & $6(15.4 \%)$ & 0.520 \\
History of prior surgery & $71(78.0 \%)$ & $45(86.5 \%)$ & $26(66.7 \%)$ & 0.039 \\
Patient presented with severe sepsis & $20(22.0 \%)$ & $10(19.2 \%)$ & $10(26.5 \%)$ & 0.610 \\
ASA score & $3.7 \pm 0.7 \%$ & $3.5 \pm 0.7$ & $4.0 \pm 0.7$ & 0.003 \\
Clinical signs of bowel movement & $59(64.8 \%)$ & $36(69.2 \%)$ & $23(59.0 \%)$ & 0.377 \\
CT with signs of ischemia/thrombus & $23(25.3 \%)$ & $10(19.2 \%)$ & $13(33.3 \%)$ & 0.148 \\
\hline
\end{tabular}

had statistically higher mean serum lactate values than the survivors (5.6 \pm 4.8 vs. $3.0 \pm 2.2 \mathrm{mmol} / \mathrm{L}, p=0.024)$.

A total of 34 patients $(37.4 \%)$ had at least two serum lactate measurements within $24 \mathrm{~h}$ before surgery. Of those, 17 patients had an increase, and 17 patients a decrease in serum lactate before laparotomy. In this subgroup of patients, an increase in serum lactate did not correlate with the length of bowel necrosis or with outcome. Serial serum C-reactive protein (CRP) and white cell counts were of no predictive value for any of the outcome parameters (data not shown).

\section{Intraoperative Parameters and Outcome}

In all of the 91 patients, an explorative laparotomy was conducted and AMI was confirmed macroscopically and histologically. Bowel ischemia involved the small bowel in
Fig. 1 Serum lactate measurements before laparotomy

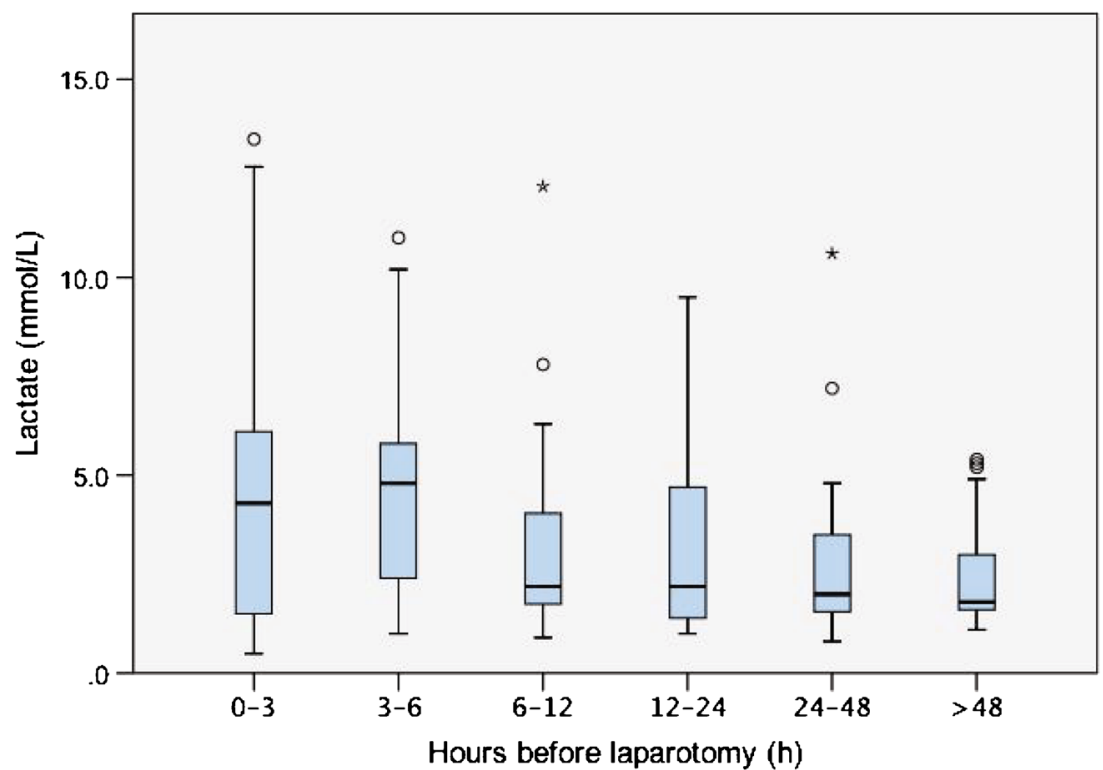


Table 2 Correlation of length of bowel necrosis and serum lactate levels

\begin{tabular}{lllll}
\hline $\begin{array}{l}\text { Length } \\
\text { of bowel } \\
\text { necrosis }(\mathrm{cm})\end{array}$ & Number & $\begin{array}{l}\text { Highest lactate } \\
0-6 \mathrm{~h} \text { before } \\
\text { surgery }(\mathrm{mmol} / \mathrm{L})\end{array}$ & $\begin{array}{l}\text { Highest lactate } \\
6-24 \mathrm{~h} \text { before } \\
\text { surgery }(\mathrm{mmol} / \mathrm{L})\end{array}$ & $p$ \\
\hline$<50$ & 40 & $3.95 \pm 2.94(n=20)$ & $3.19 \pm 2.75(n=9)$ & 0.517 \\
$>50$ & 42 & $6.30 \pm 5.36(n=20)$ & $2.58 \pm 1.74(n=11)$ & 0.034 \\
$p$ & & 0.094 & 0.553 & \\
\hline
\end{tabular}

$56.1 \%$, the large bowel in $18.7 \%$, and both the large and small bowels in $27.5 \%$ of patients. The average length of necrosis was $94.1 \pm 103.9 \mathrm{~cm}$.

The in-hospital mortality rate was $42.9 \%(n=39)$. A significantly higher ASA score was detected for the non-survivors than for the survivors $(4.0 \pm 0.7$ vs. $3.5 \pm 0.7, p=$ $0.003)$. Moreover, the operation time was significantly shorter in the non-survivor group ( $184 \pm 82$ vs. $134 \pm 89 \mathrm{~min}$, $p=0.005$ ). Furthermore, the non-survivors tended to have less intraoperative blood loss than the survivors (454 \pm 657 vs. $273 \pm 426 \mathrm{~mL}, p=0.053)$.

Ischemia of the small and large bowels combined was significantly more common in non-survivors than in survivors ( 46.3 vs. $13.5 \% ; p=0.001$ ). Forward logistic regression showed that the length of necrotic bowel and the highest lactate value within $24 \mathrm{~h}$ prior to surgery were independent risk factors for mortality $\left(r^{2}=0.329\right)$.

\section{Discussion}

Serum lactate levels are considered an aid in detecting AMI. ${ }^{9 \cdot 10}$ Unfortunately, newer studies have failed to confirm that lactate is a reliable marker and no other reliable serum markers have been identified. ${ }^{11^{-13}}$ However, only single preoperative values have been assessed. There have been no studies with serial measurements of lactic acidosis in this group of severely ill patients.

In this study, for the first time, patients with AMI were retrospectively evaluated with the aim to assess the diagnostic role of repeated preoperative serum lactate and $\mathrm{pH}$ measurements and to identify risk factors for a lethal outcome in patients

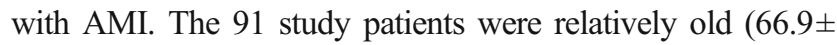
15.7) and had a high rate of known cardiovascular disease $(68.1 \%)$, which is in accordance with the literature and the evolution of the disease - with arteriosclerosis as one of the underlying pathophysiological conditions. ${ }^{9 \cdot 14}$ Among the preoperative parameters obtained, only the ASA score showed a significant difference between the survivor and non-survivor groups, being significantly higher in the non-survivor group ( $3.47 \pm 0.67$ vs. $3.97 \pm 0.72, p=0.003$ ). However, this finding is most probably not due to the patient's demographics, but rather with the advanced clinical stage of AMI when they presented.

As expected, a high percentage $(82.6 \%)$ of patients was evaluated with a CT scan before laparotomy. However, only $25.3 \%$ revealed clear signs of AMI. For the remaining patients, only suspicious signs of AMI were noted within the radiology reports. Different etiologies for bowel necrosis may be the reason for this finding.

All of the 91 patients underwent laparotomy and AMI was confirmed macroscopically and histologically. The mortality rate was $42.9 \%$. The forward logistic regression analysis revealed that the length of necrotic bowel and the highest lactate value within $24 \mathrm{~h}$ prior to surgery were independent risk factors for mortality $\left(r^{2}=0.329\right)$. On average, $94.1 \pm 103.9 \mathrm{~cm}$ of ischemic bowel was detected. In $56.1 \%$ of patients, solely, the small bowel was affected, which reflects occlusions of the superior mesenteric artery, described as the most frequent site causing AMI. ${ }^{1}$ Interestingly, patients with small and large bowel ischemia showed a significantly higher mortality rate (46.2 vs. $13.5 \%$, $p=0.001$ ) than patients with only the small or large bowel affected. This subgroup of patients with small- and large-bowel ischemia could have suffered from multiple thromboembolic events.

Operative time was significantly shorter and intraoperative blood loss was less in the non-survivor group (Table 3). This
Table 3 Intraoperative parameters and outcomes

\begin{tabular}{lllll}
\hline & All $(n=91)$ & Survivor $(n=52)$ & Non-survivor $(n=39)$ & $p$ \\
\hline Ischemia $>100 \mathrm{~cm}$ & $23(29.9 \%)$ & $10(22.2 \%)$ & $13(39.4 \%)$ & 0.136 \\
Ischemia $>50 \mathrm{~cm}$ & $39(50.6 \%)$ & $19(43.2 \%$ & $20(60.6 \%)$ & 0.169 \\
Blood loss $(\mathrm{mL})$ & $376 \pm 500$ & $454 \pm 657$ & $273 \pm 426$ & 0.053 \\
Operative time (min) & $163 \pm 88.9$ & $184 \pm 82$ & $134 \pm 89$ & 0.005 \\
Large-bowel ischemia & $17(18.7 \%)$ & $13(25.0 \%)$ & $4(10.3 \%)$ & 0.104 \\
Small-bowel ischemia & $51(56.1 \%)$ & $33(63.5 \%)$ & $18(46.2 \%)$ & 0.135 \\
Large- and small-bowel ischemia & $25(27.5 \%)$ & $7(13.5 \%)$ & $18(46.2 \%)$ & 0.001 \\
Second look laparotomy & $23(25.3 \%)$ & $11(21.2 \%)$ & $12(13.2 \%)$ & 0.336 \\
Length of bowel necrosis $(\mathrm{cm})$ & $94.1 \pm 104.0$ & $70.6 \pm 76.9$ & $127.1 \pm 127.3$ & 0.192 \\
\hline
\end{tabular}


finding is explained by the explorative laparotomies without further surgical intervention conducted in patients with generalized, fatal visceral ischemia.

In the current study, more than two serum lactate measurements were available per patient, allowing the detection of an increase or decrease in preoperative values. Interestingly, the overall mean serum lactate level at $0-6 \mathrm{~h}$ before surgery was significantly higher and the $\mathrm{pH}$ lower than in the period of more than $6 \mathrm{~h}$ before surgery. Moreover, the highest serum lactate value measured within $24 \mathrm{~h}$ before surgery in individual patients showed a moderate correlation, but no statistical significance with the length of bowel necrosis $\left(r^{2}=0.257, p=\right.$ 0.058 ). However, high variability was observed, and therefore, this finding cannot be translated to the individual patient. To further elucidate the role of serial measurements and to limit the heterogeneity of the measurements, we assessed the subgroup of patients with two or more repeated serum lactate measurements performed within the last $24 \mathrm{~h}$ prior to surgery. In this subgroup of 34 patients, 17 had a decrease and 17 an increase in serum lactate before laparotomy, which implies that lactate levels are probably influenced by multiple variables. One of the most important variables might be aggressive fluid resuscitation in deteriorating patients before surgery, as this causes a decrease in lactate values.

The observation that serum lactate might have a predictive value in the amount of bowel affected by ischemia was more robust. In the subgroup of patients with $>50 \mathrm{~cm}$ of necrotic bowel, a significant increase was found in serum lactate $0-6 \mathrm{~h}$ before surgery compared to 6-24 h before surgery. This observation was not statistically significant in those patients with $<50 \mathrm{~cm}$ of necrotic bowel. Moreover, a trend towards higher serum lactate $0-6 \mathrm{~h}$ before surgery was seen in patients with $>50 \mathrm{~cm}$ ischemia compared to patients with $<50 \mathrm{~cm}$ ischemia. Once again, these results were highly variable and the number of patients was limited, so that diagnostic interpretation or cutoff analysis is impossible. ${ }^{15}$

The limitations of this study are its retrospective design and the relatively small number of patients, especially of those patients with $\geq 2$ lactate measurements within $24 \mathrm{~h}$ prior to surgery. The measurements of lactacidosis were at the discretion of the attending physician causing a wide variability of the timing of the blood tests. Furthermore, the retrospective nature of this study and limited number of patients did not allow to accurately differentiate between the causes of bowel necrosis or a comparison between subgroups. Therefore, the conclusions were drawn with caution.

\section{Conclusion}

AMI remains a serious surgical emergency with high mortality. The length of necrotic bowel and the highest lactate value within $24 \mathrm{~h}$ prior to surgery are independent risk factors for mortality. However, serum lactate and $\mathrm{pH}$ are of limited value, even when measured repeatedly, as multiple variables probably influence the level of lactacidosis in this group of severely ill patients. To the best of our knowledge, the evaluation of serial serum lactate levels is new to the literature. In this cohort, the serial lactate measurements added no benefits in the evaluation of severely ill patients with AMI.

\section{References}

1. Acosta S. Epidemiology of mesenteric vascular disease: clinical implications. Semin Vasc Surg 2010;23:4-8.

2. Kairaluoma MI, Karkola P, Heikkinen D, Huttunen R, Mokka RE, Larmi TK. Mesenteric infarction. Am J Surg 1977;133: 188-193.

3. Kortmann B, Klar E. Recognizing acute mesenteric ischaemia too late: reasons and diagnostic approach from a surgical point of view. Zentralbl Chir 2005;130:223-226.

4. Luther. Intestinale Durchblutungsstörungen. Mesenterialinfarkt, Angina abdominalis, Therapieoptionen, Prognosen. Steinkopff Verlag Darmstadt 2001

5. Stoney RJ, Cunningham CG. Acute mesenteric ischemia. Surgery 1993;114:489-490.

6. Klein HM, Lensing R, Klosterhalfen B, Tons C, Gunther RW. Diagnostic imaging of mesenteric infarction. Radiology 1995;197: 79-82.

7. Menke J. Diagnostic accuracy of multidetector CT in acute mesenteric ischemia: systematic review and meta-analysis. Radiology 2010;256:93-101.

8. Lock G. Acute intestinal ischaemia. Best Pract Res Clin Gastroenterol 2001;15:83-98.

9. Lange H, Toivola A. Warning signals in acute abdominal disorders. Lactate is the best marker of mesenteric ischemia. Lakartidningen 1997;94:1893-1896.

10. Murray MJ, Gonze MD, Nowak LR, Cobb CF. Serum D(-)-lactate levels as an aid to diagnosing acute intestinal ischemia. Am J Surg 1994; 167:575-578.

11. Evennett NJ, Petrov MS, Mittal A, Windsor JA. Systematic review and pooled estimates for the diagnostic accuracy of serological markers for intestinal ischemia. World J Surg 2009;33:1374-1383.

12. Block T, Nilsson TK, Bjorck M, Acosta S. Diagnostic accuracy of plasma biomarkers for intestinal ischaemia. Scand J Clin Lab Invest 2008;68:242-248.

13. Acosta S, Nilsson T. Current status on plasma biomarkers for acute mesenteric ischemia. Journal of thrombosis and thrombolysis 2012;33:355-361.

14. Gearhart SL, Delaney CP, Senagore AJ, Banbury MK, Remzi FH, Kiran RP, Fazio VW. Prospective assessment of the predictive value of alpha-glutathione S-transferase for intestinal ischemia. Am Surg 2003;69:324-329; discussion 329.

15. Acosta S, Block T, Bjornsson S, Resch T, Bjorck M, Nilsson T. Diagnostic pitfalls at admission in patients with acute superior mesenteric artery occlusion. J Emerg Med 2012;42:635641. 